\title{
Microzonificación sísmica basada en efectos de sitio para el área metropolitana de Mendoza
}

\section{Sismic microzonation based on site effects for the metropolitan area of Mendoza}

Presentación: 06-07/10/2020

\author{
Doctorando: \\ Emilce Gisela Giolo \\ CeReDeTEC (Centro Regional de Desarrollos Tecnológicos para la Construcción, Sismología e Ingeniería sísmica), Facultad Regional \\ Mendoza, Universidad Tecnológica Nacional - Argentina. \\ emilce.giolo@frm.utn.edu.ar
}

\section{Director/a:}

\section{Carlos Daniel Frau}

\section{Co-directores:}

\section{Bertrand Guillier \\ Miguel Eduardo Tornello}

\section{Resumen}

El ordenamiento territorial de una región debe contemplar distintos aspectos que hacen a las características del entorno y al hábitat humano inserto en ella. Algunos de los peligros potenciales asociados a la actividad sísmica son la licuación de suelos, deslizamiento de tierras, caída de rocas, efectos de sitio debido a terremotos, variaciones topográficas, tsunamis entre otros. En particular la respuesta de sitio puede ser evaluada por métodos teóricos o empíricos. Los métodos teóricos permiten un análisis más detallado de los diferentes estratos de suelo y los métodos empíricos se basan en el registro de vibraciones producidas por terremotos u otras causas en sitios de diferentes condiciones geológicas. Entre los métodos experimentales para considerar efectos locales, han tenido gran aceptación en las últimas décadas los basados en vibraciones ambientales. En el presente trabajo se escoge un área de gran densidad poblacional en la Ciudad de Mendoza, Argentina para realizar una microzonificación sísmica en base a frecuencias naturales de suelo que se obtienen mediante el procedimiento del cociente espectral H/V. De esta manera se pretende identificar las áreas expuestas al fenómeno de amplificación sísmica cuando sobrevenga un terremoto de mediana o elevada magnitud. Este estudio permitirá contar con información de utilidad para la organización del uso del suelo y la planificación urbana.

Palabras clave: Microzonificación sísmica - frecuencia del suelo - efecto de sitio.

\begin{abstract}
The territorial ordering of a region must contemplate different aspects that make the characteristics of the environment and the human habitat inserted in it. Some of the potential hazards associated with seismic activity are soil liquefaction, landslide, rockfall, site effects due to earthquakes, topographic variations, tsunamis, among others. The site response can be evaluated by theoretical or empirical methods. The theoretical methods allow a more detailed analysis of the different soil layers and the empirical methods are based on the recording of vibrations produced by earthquakes or other causes, in sites of different
\end{abstract}


geological conditions. Among the experimental methods for considering local effects, those based on ambient vibrations have been widely accepted in recent decades. In the present paper, an area of high population density is selected in the city of Mendoza, Argentina, to perform a seismic microzoning based on natural soil frequencies obtained by the H/V spectral ratio procedure. In this way the areas exposed to the phenomenon of seismic amplification are identified when a medium or high magnitude earthquake occurs. This microzoning will provide useful information for the organization of land use and urban planning.

Keywords: Seismic Microzonation - Frecuency of Soil - Site Effect,

\section{Introducción}

El ordenamiento territorial de una región debe contemplar distintos aspectos que hacen a las características del entorno y al hábitat humano inserto en ella. Entre los aspectos a considerar se encuentran los riesgos naturales. Si la región geográfica en cuestión es una zona tectónicamente activa, deben contemplarse los múltiples aspectos relacionados con la ocurrencia de terremotos. La identificación de áreas con diferentes potenciales de amenaza sísmica es necesaria para el ordenamiento territorial de aquéllas ciudades cercanas a fuentes sísmicas en orden a reducir el riesgo y la vulnerabilidad de los edificios y de las obras de infraestructura. Algunos de los peligros potenciales asociados a la actividad sísmica son la licuación de suelos, deslizamiento de tierras, caída de rocas, efectos de sitio debido a terremotos, variaciones topográficas, tsunamis entre otros.

Es bien conocido que las condiciones y efectos locales de un sitio son un factor de importancia en la respuesta de suelos blandos ante terremotos y que este aspecto necesita ser considerado adecuadamente (Bendimerad, F. 2003). Desde hace ya varias décadas que la inclusión de efectos de sitio en los códigos de diseño sísmico ha recibido gran atención por parte de la comunidad ingenieril (Chavez-García y Faccioli 2004, Adams y Atkinson 2003).

La respuesta de sitio puede ser evaluada por métodos teóricos o empíricos. Entre los métodos experimentales para considerar efectos locales, han tenido gran aceptación en las últimas décadas los basados en microtremores o vibraciones ambientales. Mediante el análisis de las mediciones de vibraciones ambientales se puede determinar el período fundamental de oscilación cuando existe un contraste de impedancia importante entre los estratos que conforman el depósito sedimentario (Lermo y Chavez-García 1994).

Entre las diferentes técnicas que utilizan microtremores, el método de las relaciones espectrales $\mathrm{H} / \mathrm{V}$ conocido como método de Nakamura (Nakamura 1989, Nakamura 2000) ha alcanzado una gran aceptación por lo que ha sido aplicado en un gran número de regiones diferentes en todo el mundo. Entre otras razones, su amplia difusión se debe a que sólo requiere analizar los datos registrados por una estación de tres componentes emplazada en la superficie del sitio a auscultar.

La sismicidad de Argentina es consecuencia de la convergencia de las placas Sudamericana y de Nazca; dentro de este marco, el piedemonte andino de la Provincia de Mendoza reúne una buena parte de la deformación tectónica reciente asociada al crecimiento de la Cordillera de los Andes que se manifiesta por la concentración de la sismicidad (Bastías et al. 1993, Modaressi et al. 1998). Los epicentros de los sismos registrados indican que la mayor parte de la actividad sísmica se concentra en el centro-oeste de nuestro país caracterizando a la región como de "elevada actividad sísmica". Esta elevada sismicidad puede conjugarse con características particulares del subsuelo provocando efectos de sitio, esto es amplificación de las ondas sísmicas debido a la baja rigidez del sustrato que media entre la superficie del suelo y el basamento. La exposición a este tipo de peligros es particularmente relevante para centros urbanos e infraestructuras críticas, por las potenciales pérdidas de vidas humanas y bienes que pueden resultar de ello.

En el presente trabajo se escoge el área metropolitana de la provincia de Mendoza, Argentina para realizar una microzonificación sísmica en base a frecuencias naturales de suelo que se obtienen mediante el procedimiento del cociente espectral H/V. De esta manera se identifican las áreas expuestas al fenómeno de amplificación sísmica cuando sobrevenga un terremoto de mediana o elevada magnitud. Esta microzonificación permitirá contar con información de utilidad para la organización del uso del suelo y la planificación urbana. 


\section{Desarrollo}

\section{Efectos de Sitio}

Se conoce como efecto de sitio a la respuesta sísmica del terreno con características significativamente distintas en amplitud, duración o contenido de frecuencias de un área relativamente reducida, con respecto al entorno regional. En otras palabras, podría decirse que el efecto de sitio es la transformación que sufren las ondas sísmicas desde la roca base hasta la superficie, pudiendo variar la amplificación de la onda en varios órdenes de magnitud.

La naturaleza y la distribución de los daños que se pueden producir en las estructuras con la ocurrencia de un terremoto, está muy influenciada por la respuesta del terreno frente a cargas cíclicas, como consecuencia de lo cual se produce una modificación de la señal sísmica (Aki, K. 1998). El estudio de la respuesta dinámica del suelo en el análisis de riesgo sísmico y en el diseño sismorresistente está siendo objeto de numerosos trabajos de investigación, sobre todo luego de los recientes terremotos ocurridos en el mundo (Northbridge, 1994; Kobe, 1995; Turquía, 1999; El Salvador, 2001; Chile, 2010; Nepal, 2015; Ecuador, 2016).

La presencia de terrenos, considerados geotécnicamente como blandos, causa un aumento considerable de los daños generados por terremotos en todo tipo de estructuras situadas sobre ellos. La respuesta y la magnitud de la amplificación de la señal están controladas principalmente por las propiedades de los materiales geológicos, especialmente por sus características dinámicas como, las velocidades de corte, el espesor de las capas, la profundidad a la que se encuentra el sustrato rocoso, y finalmente la diferencia de las Vs entre roca sísmica $( \pm 800 \mathrm{~m} / \mathrm{s})$ y sedimentos (Aki, K. 1998, Borcherdt, R. D. 1970).

\section{Metodología}

El método H/V consiste en la utilización de microtremores para la determinación de efectos de sitio a través de la frecuencia natural, mediante la realización de un cociente entre los espectros de Fourier de las componentes horizontales y verticales de un registro en el sitio estudiado. Se estima que los microtremores contienen predominantemente ondas $\mathrm{S}$, por lo que la relación espectral $\mathrm{H} / \mathrm{V}$ es igual a la función de transferencia de dichas ondas entre la superficie y la base de la capa sedimentaria; así, el pico del espectro resultante es el correspondiente a la frecuencia fundamental del sitio. De esta manera, la excitación de la capa superficial provoca la amplificación del efecto de sitio producto de las ondas S. Por otro lado, las ondas Rayleigh que se propagan en las capas sedimentarias sobre lechos rocosos provocan el mismo efecto en las componentes horizontales y verticales del movimiento en la superficie. Esta metodología trata de eliminar el efecto que producen las ondas Rayleigh, de esa manera la función de transferencia queda determinada por ondas de cuerpo S. Simplificadamente se plantea un modelo en el cual un suelo sedimentario está apoyado sobre un estrato rocoso donde la principal diferencia entre estos estratos es la velocidad de la onda S. Para el desarrollo del procedimiento, se plantea una serie de relaciones entre las amplitudes de los espectros de Fourier de las distintas componentes del movimiento tanto en superficie como en el lecho rocoso. Siendo $\mathrm{Hb}$ y $\mathrm{Vb}$, las amplitudes espectrales del movimiento horizontal y vertical en el lecho rocoso respectivamente, y Hs y $V_{S}$ las amplitudes espectrales de las componentes horizontal y vertical del movimiento en superficie. Considera que en el lecho rocoso la propagación de las ondas va a ser la misma en todas las direcciones $\mathrm{Hb}(\omega)=\mathrm{Vb}(\omega)$ se llega a la ecuación (1). Se puede estimar el efecto de sitio mediante $\mathrm{S}_{\mathrm{M}}(\omega)$, la ecuación (1) es conocida como Espectro de Cuasi Transferencia (QTS).

$$
S_{M}(\omega)=\frac{H_{s}(\omega)}{V_{S}(\omega)}
$$

Las mediciones se realizaron con acelerómetros modelo Basalt de Kinemetrics. Poseen un sensor triaxial interno (Epi Sensor Force Balance) y se alimentan con una batería de $12 \mathrm{~V}$. Los acelerómetros fueron configurados para medir de forma continua, almacenando los registros cada 10 minutos, con una frecuencia de muestreo de $200 \mathrm{~Hz}$. En este estudio se han seguido las directrices dada por el manual del proyecto SESAME (European Research Program, 2004) y el software Geopsy para el procesamiento de señales. 
Área de estudio

El área de estudio considerada encierra el área metropolitana de Mendoza, ésta incluye la zona más comercial de la Ciudad de Mendoza, administrativa y Centro Cívico, incluye también zonas residenciales de distintas jerarquías con edificios de altura. Comprende también el área netamente comercial del Departamento de Godoy Cruz, fundamentalmente en torno a los ejes de calle San Martín Sur y calle Brasil; el área residencial, se desarrolla en todo el Departamento, pero se halla de forma conjunta con la comercial; en este aspecto se incluye francamente a la zona de Villa Marini, la cual sufriera los mayores daños durante el terremoto de Mendoza de 1985. En cuanto al Departamento de Las Heras se contempla el área cívico y más comercial en su proximidad con la Ciudad Capital, donde también se produjeron importantes daños en el terremoto de 1985. En la figura 1 se observa el área Metropolitana de Mendoza de acuerdo al plan de ejecución Metropolitana del programa DAMI efectuado en el año 2013.

Dentro del área Metropolitana se ha definido una grilla con los puntos a medir, la misma se ha realizado con una separación de $1000 \mathrm{~m}$ en toda la extensión, obteniendo un total de 298 puntos a estudiar (Figura 2).

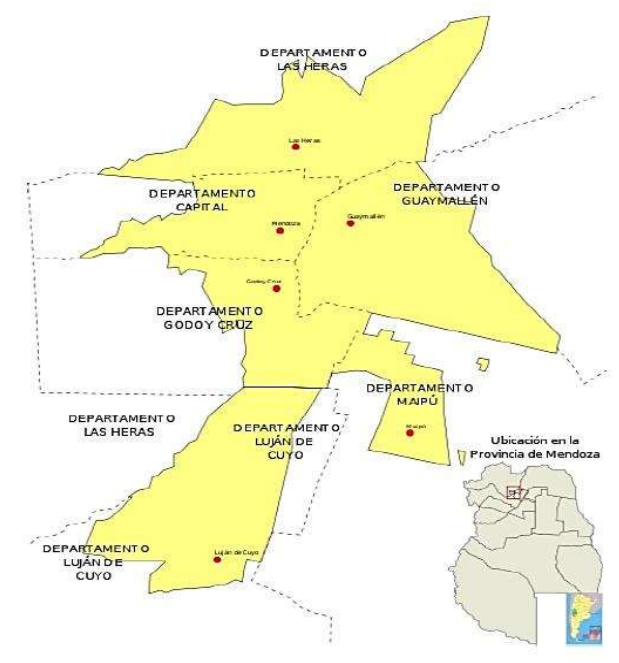

Figura 1: Área Metropolitana de Mendoza a estudiar

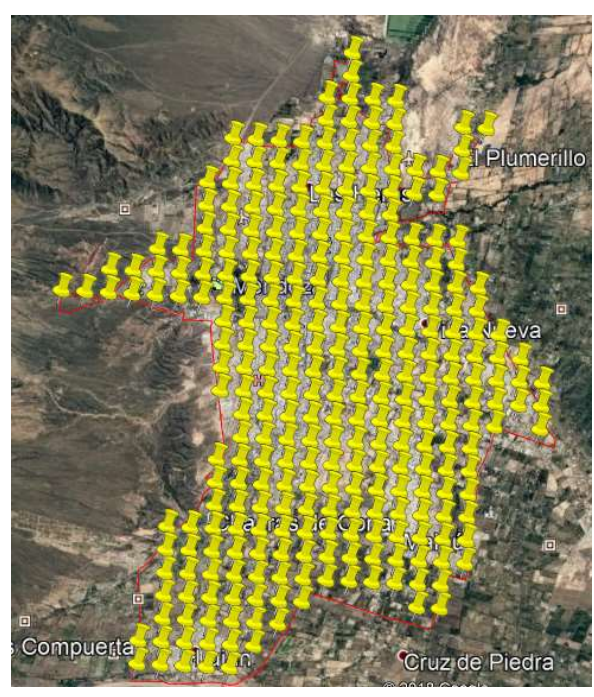

Figura 2: Puntos a medir obtenidos mediante grilla con pasos cada $1000 \mathrm{~m}$.

\section{Resultados}

En el presente apartado se vuelcan los resultados de las mediciones realizadas para obtener las frecuencias naturales $\mathrm{f}_{0}$ en cada uno de los sitios auscultados o bien su período natural $\mathrm{T}_{0}=1 / \mathrm{f}_{0}$ obtenidos a partir de mediciones de vibración ambiental y aplicando el método de Nakamura. Unas síntesis de los resultados de los estudios realizados hasta el momento se presentan en el mapa de la Figura 3 (izquierda), donde se muestran las frecuencias del suelo obtenido con los resultados de algunos puntos medidos dentro del área estudiada (Giolo et al. 2018). En dicho mapa puede observarse una zona de altas frecuencias hacia el sudoeste. Se identifica claramente visible una línea, que atraviesa el Gran Mendoza de Nor-Este a Sud-Oeste, donde las frecuencias cambian abruptamente de un lado y del otro de dicha línea. Esta anomalía puede entenderse como la existencia de cambios en las condiciones del subsuelo. Este cambio puede originarse como fruto de la variación de algunos parámetros tales como la velocidad de onda de corte y/o el espesor del sedimento. Puede notarse además una variación marcada de frecuencias, en dos zonas del departamento de Godoy Cruz, Villa Hipódromo y Villa Marini, dichas zonas se muestran en forma de islas. La figura 3 (derecha) presenta un mapa de Intensidad Mercalli Modificada (IMM) correspondiente al terremoto de Mendoza de 1985. Puede notarse que las zonas de Villa Marini y Villa Hipódromo sufrieron IMM VIII, las cuales coinciden con las zonas de altas frecuencias que se pueden visualizar en el mapa de la izquierda. En la zona norte de la ciudad de Mendoza y la villa 
cabecera del departamento de Las Heras, se identificaron frecuencias más bajas mostrando coincidencia con la zona de mayores daños en el terremoto de 1985.

Investigaciones recientes (ej.: Hellel et al. 2012) han utilizado el método de Nakamura como complemento en la investigación de fallamientos y profundidad de estratos hasta el lecho rocoso. Considerando que existe una relación teórica entre la velocidad de la onda de corte Vs y el periodo o frecuencia del suelo f, donde la Vs representa en cierta medida la rigidez de los estratos que atraviesa, es decir que suelos más rígidos tendrán velocidades mayores que suelos menos rígidos, y que adicionalmente la profundidad del estrato nos da una idea de la masa involucrada se podrían interpretar estas variaciones bruscas de frecuencia como: 1) los estratos a un lado y al otro de la línea tienen velocidades de onda de corte diferentes, y 2) Exista una variación del estrato de suelo sísmicamente activo, siendo de mayor profundidad en la zona al Oeste de la línea de trazo (frecuencias bajas) y de menor profundidad en la zona al Este de la línea de trazo (altas frecuencias). Esta última situación podría poner de manifiesto la presencia de una falla ciega inversa con vergencia al oeste, similar a las del sistema de falla del Cerro de La Gloria aflorantes $3 \mathrm{~km}$ al oeste. Esta hipótesis debe comprobarse con la ayuda de estudios complementarios.

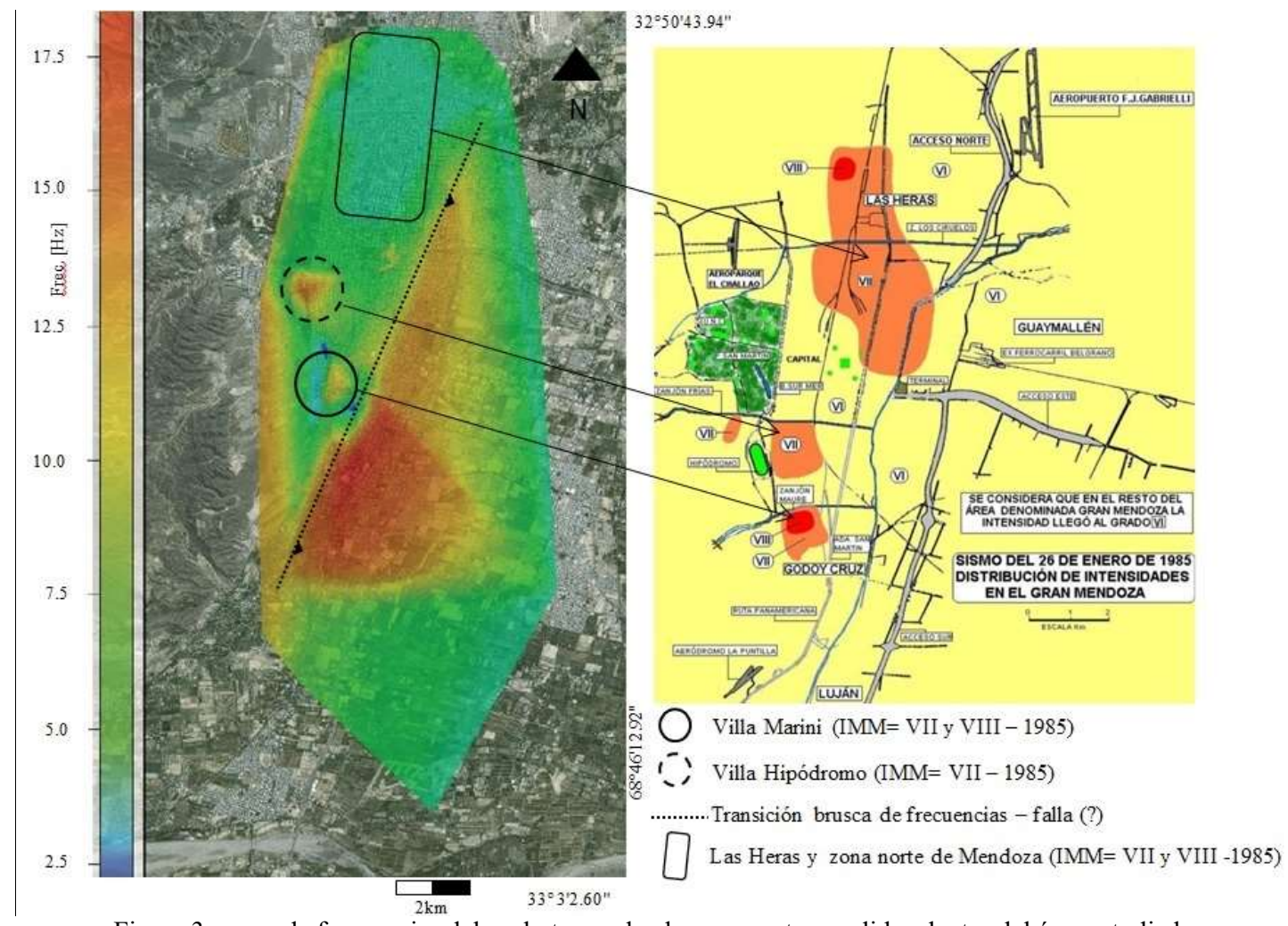

Figura 3: mapa de frecuencias del suelo tomando algunos puntos medidos dentro del área estudiada.

\section{Conclusiones}

En el área de estudio las frecuencias naturales de suelo encontradas presentan variaciones considerables que van desde los 2 $\mathrm{Hz}$, suelos más blandos desde el punto de vista del diseño sismorresistente de estructuras, a los $18 \mathrm{~Hz}$ aproximadamente, siendo estos suelos más rígidos. En el mapa de la figura 3 (derecha) se observan dos islas correspondientes a la zona de Villa Marini (coincidente con las amplificaciones encontradas en los registros sísmicos) y Villa Hipódromo en el departamento de Godoy Cruz, Mendoza. En dichas islas las frecuencias obtenidas van desde los $2 \mathrm{~Hz}$ en los bordes a $18 \mathrm{~Hz}$ hacia el centro de las mismas. La presencia de estas variaciones indican ciertas perturbaciones en el sub-suelo, que podrían provocar mayores intensidades durante sismos locales, tal como ocurrió en dichas zonas durante el sismo del 25 de enero de 1985 en Mendoza. Se observa además que las frecuencias van en orden creciente hacia la zona del piedemonte, lo cual se corresponde con el sustrato más rígido tal como el que posee dicha zona. 
La variación de frecuencias detectada a lo largo de un eje que atraviesa el Gran Mendoza de Nor-Este a Sud-Oeste puede ser interpretada como la presencia de una falla inversa ciega. A partir de resultados de investigaciones previas, se detecta que la técnica del coeficiente espectral $\mathrm{H} / \mathrm{V}$ puede capturar variaciones del sustrato por lo que puede ser aplicada para identificar estructuras ciegas, en particular fallas o estructuras potencialmente sismogénicas (Giolo et al., 2019).

\section{Referencias}

Adams, J., y G. Atkinson, 2003. Development of seismic hazard maps for the proposed 2005 edition of National Building Code of Canada. Journal of Civil Engineering: 30:255-71.

Aki, K. (1988). Local site effects on ground motion, in Earthquake. Engineering and Soil Dynamics. Recent Advances in Ground Motion Evaluation, J. L. Von Thun (Editor), Geotechnical Special Publication No. 20, Am. Soc. Civil Eng., New York, pp. 103- 155.

Bastías, H., G. Perucca, y J. Paredes 1993. Peligro sísmico y neotectónica. En XII Congreso Geológico Argentino Relatorio, de V. Ramos, 645-658.

Bendimerad, F. 2003. The Boumerdes, Argelia, Earthquake of May 21, 2003. EERI Learning from Earthquakes Reconnaissance.

Borcherdt, R. D. (1970). Effects of local geology on ground motions near San Francisco Bay. En: Bull. Seism. Soc. Am. Vol. 60 , pp. $29-61$.

Chavez-García, F. J., y Faccioli E. 2004. Complex site effects and building code: making the leap. Seismol J.: 4(1):23-40.

Di Alessandro, C., Bonilla L. F., Boore D. M., Rovelli A. y Scotti O. 2012. Predominant-Period Site Classification for Response Spectra Prediction Equations in Italy. Bull. Seismol. Soc. Am. 102, nº 2: 680-695.

Giolo, E., Calderón, F., Pagano, S., Vía Gatica, S., Frau C. (2019). Revista de Geología Aplicada a la Ingeniería y al Ambiente $\mathrm{N}^{\circ} 43$, pag. $55-67$ (2019).

Giolo, E., Frau, C., Calderón, F., Pagano, S., Tornello, M. (2018). Frecuencias naturales de suelos en el núcleo urbano de la ciudad de Mendoza. Revista Internacional de Ingeniería de Estructuras Vol. 23,2, 157 - 188 (2018), doi: 10.24133/riie.v23i2.967.

Hellel, M., Oubaiche, E. H., Chatelain, J. L., Machane, D., Bensalem, R., Guillier, B. and Cheikhlounis, G. 2012. Basement Mapping with Single-Station and Array Ambient Vibration Data: Delineating Faults under Boumerdes City, Algeria. Seismological Research Letters Volume 83, Number 5 September/October 2012. doi: 10.1785/0220110142.

Lermo, J., y Chavez-García, F. 1994. Are microtremors useful in site response evaluation? Bulletiin of Seism. Soc. American, San Francisco: 1350-64.

Modaressi, H., Mellal, A., y Bour, M. 1998. Evaluation of seismic response spectra using a unified numerical approach. Proceedings of the 11th European Conference on Earthquake Engineering. Amsterdam: Balkema.

Nakamaura, Y. 1989. A method for dynamic characteristic estimation of subsurface using microtremors on the ground surface. Quartely Report of Railway Technical Research Institute 30: 25-33.

Nakamura, Y. 2000. Clear identification of fundamental idea of Nakamura's technique and its applications. Proceedings of the 12th World Conference on Earthquake Engineering. Auckland, New Zealand. Paper 2656.

SESAME European Research Program 2004. Guidelines for the implementation of the H/V spectral ratio technique on ambient vibrations, measurements, processing and interpretation. 